\title{
Potencial da microalga Dunaliella Tertiolecta BUTCHER na geração de biocombustíveis e biorremediação de efluente de reator anaeróbio
}

A microalga Dunaliella tertiolecta BUTCHER apresenta-se como possível fonte de biomassa para produção de biocombustíveis, se destacando devido ao seu potencial de produção lipídica. Com o objetivo de otimizar o desenvolvimento celular da microalga e verificar sua potencialidade na produção de lipídios, utilizouse o meio de cultura sintético $\mathrm{F} / 2 \mathrm{com} \mathrm{pH}$ distintos $(5,5 ; 6,5 ; 7,23 ; 8,5$ e 9,5) suplementado com efluente de reator do tipo UASB (nas proporções $0,25,50,75$ e $100 \%$ ) em quatro intervalos de exposição luminosa $(6,12,18$ e 24 horas). Percebeu-se uma melhor resposta de crescimento celular da D. tertiolecta BUTCHER quando submetida a intervalos de 12 horas de exposição à luz, em pH 7,23 com uma concentração de efluente de UASB numa proporção de $25 \%$, verificando-se, nessas condições, uma remoção de $40,23 \%$ de DQO. Em relação a concentração de lipídios, foi obtido para o cultivo da microalga em meio $\mathrm{F} / 2$ um teor lipídico de $10,64 \%$, enquanto a cultivada com $25 \%$ de efluente do reator UASB apresentou 8,13\%. A D. tertiolecta BUTCHER quando cultivada em meio alternativo apresenta um melhor perfil para biorremediação ao comparar com o seu potencial para geração de biocombustíveis, verificado a partir da redução do teor lipídico.

Palavras-chave: Biomassa; UASB; Otimização.

\section{Potential of the microalga Dunaliella Tertiolecta BUTCHER to generate biofuels and bioremremediation of effluent from anaerobic reactor}

\begin{abstract}
The microalgae Dunaliella tertiolecta BUTCHER is presented as a possible source of biomass for biofuel production, especially due to its potential for lipid production. Aiming to optimize the cellular development of this microalgae in order to verify its potentiality in the production of lipids, $\mathrm{F} / 2$ synthetic medium with diverse $\mathrm{pH}(5,5 ; 6,5 ; 7,5 ; 8,5$ and 9,5) supplemented with effluent of reactor type UASB (in the following proportions: $0,25,50,75$ and $100 \%)$ was used, using four different luminosity intensities $(6,12,18$ and 24 hours). It was perceived a better cell growth response of D. Tertiolecta BUTCHER when subjected to intervals of 12 hours of exposure to light, at PH 7.23 with a concentration of effluent of UASB in a proportion of $25 \%$, verifying, under these conditions, a removal of $40.23 \%$ of COD. Regarding the lipid concentration, a lipid content of $10.64 \%$ was obtained from this microalgae cultivation in F/2 medium and $8.13 \%$ lipid content when cultivated with $25 \%$ effluent of the UASB reactor. D. tertiolecta BUTCHER, when grown in an alternative culture medium presents a better bioremediation profile compared to its biofuel generation potential, verified from the reduction of lipid content.
\end{abstract}

Keywords: Biomass; UASB; Optimization.

Topic: Engenharia Sanitária

Reviewed anonymously in the process of blind peer.
Received: 07/05/2020

Approved: 04/06/2020
Whelton Brito dos Santos (iD)

Universidade Federal de Campina Grande, Brasil

http://lattes.cnpq.br/6407528093074339

http://orcid.org/0000-0002-2956-8260

wheltonbrt@gmail.com

Weruska Brasileiro Ferreira (iD

Universidade Estadual da Paraíba, Brasil

http://lattes.cnpq.br/5630172788119332

http://orcid.org/0000-0003-2381-3883

weruska brasileiro@yahoo.com.br

Natálya Ferreira Silva

Universidade Estadual da Paraíba, Brasil

http://lattes.cnpq.br/5803701479504795

http://orcid.org/0000-0001-6419-8878

natalya.esa2@gmail.com

\author{
Emanuel Júnior Silva Soares (iD \\ Universidade Estadual da Paraíba, Brasil \\ http://lattes.cnpq.br/5093205416322233 \\ http://orcid.org/0000-0002-9224-2079 \\ emanuel.junior.902@gmail.com \\ Amanda Laurentino Torquato \\ Universidade Federal de Campina Grande, Brasil \\ http://lattes.cnpq.br/9586107560324398 \\ http://orcid.org/0000-0002-1079-1315 \\ amanda.torquato02@gmail.com \\ Vera Lucia Antunes de Lima \\ Universidade Federal de Campina Grande, Brasil \\ http://lattes.cnpq.br/5379077061489077 \\ http://orcid.org/0000-0001-7495-6935 \\ antuneslima@gmail.com
}

\section{Referencing this:}

SANTOS, W. B.; FERREIRA, W. B.; SILVA, N. F.; SOARES, E. J. S.;

TORQUATO, A. L.; LIMA, V. L. A.. Potencial da microalga Dunaliella

Tertiolecta BUTCHER na geração de biocombustíveis e biorremediação de efluente de reator anaeróbio. Revista Ibero Americana de Ciências Ambientais, v.11, n.4, p.191-204, 2020. DOI:

http://doi.org/10.6008/CBPC2179-6858.2020.004.0017 


\section{INTRODUÇÃO}

A demanda por fontes de energia continua aumentando com o desenvolvimento econômico, sendo os combustíveis fósseis a principal matriz energética desde a Primeira Revolução Industrial, com o surgimento das máquinas a vapor movidas à carvão mineral. $\mathrm{O}$ advento da Segunda Revolução Industrial difundiu o uso do petróleo e seus derivados como a principal fonte de energia mundial disponível. 0 crescimento energético anda alinhado com o crescimento econômico, obrigando os países a aumentar sua capacidade de produção, transmissão e distribuição de energia, a fim de garantir sua segurança energética, principalmente em países com economias emergentes, como o Brasil (PAREDES et al., 2017).

Em busca de satisfazer a demanda do mercado, surgiram formas alternativas de produção de energia, a exemplo dos biocombustíveis, que são produzidos a partir da transesterificação de óleos presentes na biomassa vegetal, emitindo menores quantidades de dióxido de carbono $\left(\mathrm{CO}_{2}\right)$ e de partículas poluentes ao ambiente, além de serem combustíveis renováveis (MASSON et al., 2015). No entanto, existem dificuldades e desvantagens relacionadas à aplicabilidade desse tipo de combustível que estão associadas as tecnologias de obtenção e condições da agricultura, como a necessidade de grandes áreas de produção em zonas agricultáveis (CREMONEZ et al., 2015).

Nesse cenário, as microalgas se apresentam como uma nova rota de produção de biocombustíveis, devido sua potencialidade de converter o $\mathrm{CO}_{2}$ atmosférico em uma gama de metabólitos e produtos que incluem proteínas, polissacarídeos, hidrogênio e lipídeos (NASCIMENTO et al., 2016). Sua viabilidade se deve principalmente à facilidade de cultivo, quantidade de lipídios produzidos, aumento de biomassa em curtos períodos e fácil manipulação dos seus parâmetros de cultivo.

Estima-se que existam entre 70.000 a um milhão de espécies, embora apenas 44.000 destas foram analisadas (NEOFOTIS et al., 2016). Neste grupo, destaca-se a espécie Dunaliella tertiolecta BUTCHER, microrganismo fotossintético que é capaz de produzir simultaneamente grandes quantidades de carotenoides e lipídios em determinadas condições de estresse ambiental, como luminosidade e concentração salina do meio de cultivo (FRÉ et al., 2014).

Todavia, alterações nas condições ambientais podem diminuir a velocidade de desenvolvimento das células estudadas, sendo necessário a determinação das melhores condições para o crescimento da Dunaliella tertiolecta BUTCHER voltado para produção de lipídios. O cultivo de microalgas em larga escala ainda não é considerado economicamente viável, uma vez que os custos de produção e de recuperação da biomassa algal são elevados quando comparados a outros cultivos (FRANCISCO, 2016).

Umas das alternativas para minimizar os custos no cultivo das microalgas é utilização de efluente de reatores anaeróbios, neste caso o Upflow Anaerobic Sludge Blanket (UASB), incrementando-o ao meio de cultivo. Esses reatores se baseiam na decomposição anaeróbia da matéria orgânica, gerando efluentes que ainda possuem demanda química de oxigênio residual acima do recomendado pela legislação, assim como altos níveis de fósforo e nitrogênio (ALMEIDA et al., 2018). Sendo assim, as microalgas podem utilizá-los para seu desenvolvimento, removendo-os por absorção e transformando-os em biomassa na fotossíntese, 
diminuindo os custos de desenvolvimento das microalgas para fins de geração de energia.

Além de reduzir os custos da produção, a utilização de águas residuais como meio alternativo de cultivo para microalgas, torna-se uma alternativa viável para o tratamento de efluentes altamente poluidores, podendo incluir a este processo o conceito de reuso da água (PITTMAN et al., 2011). Neste contexto, o presente estudo tem como objetivo otimizar as condições de desenvolvimento da microalga Dunaliella tertiolecta BUTCHER, avaliando o comportamento celular diante as variações de parâmetros como luminosidade, potencial hidrogeniônico e diferentes concentrações de efluente de UASB no meio de cultivo, a fim de avaliar a capacidade dessa microalga na produção de biocombustíveis e seu potencial de biorremediação em rejeitos líquidos.

\section{METODOLOGIA}

\section{Cultivo da microalga Dunaliella tertiolecta BUTCHER}

A cepa da microalga Dunaliella tertiolecta BUTCHER (Figura 1A), analisada no período 192 horas (08 dias consecutivos), foi cultivada em Erlenmeyers de $500 \mathrm{~mL}$ acoplados a aeradores que favorecem homogeneização e oxigenação do sistema contendo o meio de cultura F/2, desenvolvido por Guillard (1975). Realizando os experimentos em triplicata, durante a cultivação se manteve a temperatura de $26 \pm 2^{\circ} \mathrm{C}$ e utilizou lâmpadas com potência fixa de $40 \mathrm{~W}$ para aclimação dos cultivos, conforme apresentado na Figura $1 \mathrm{~B}$.

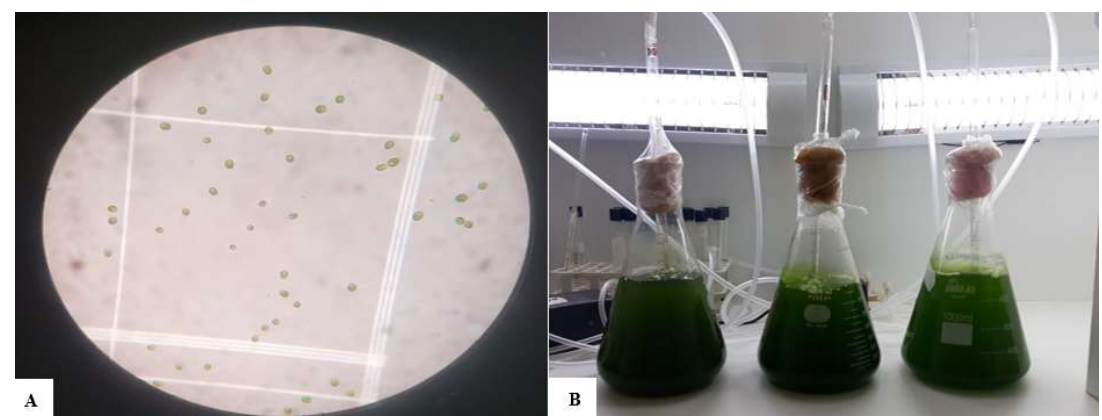

Figura 1: Fotomicrografia da microalga Dunaliella tertiolecta (A); Aclimatação dos cultivos(B).

\section{Variação das condições de cultivo}

O cultivo foi realizado em 4 etapas. Na primeira etapa, analisou-se a influência da luminosidade, submetendo os cultivos a intervalos de 6, 12, 18 e 24 horas de luz diária, determinando-se um intervalo ótimo de exposição luminosa. As culturas foram iniciadas sem alteração no pH do meio F/2, aferido em 7,23. Obtendo-se o período de iluminação ideal, verificou-se o desenvolvimento da biomassa algal em meios com $\mathrm{pH}$ distintos, mantendo o melhor intervalo de luminosidade. Nessa segunda etapa variou-se o potencial hidrogeniônico do meio entre 5,5 e 9,5, com escalonamento de 1,0. Além da análise realizada no pH característico do F/2 (Tabela 1).

Na etapa de suplementação com efluentes de UASB foram feitas diluições de 0, 25, 50, 75 e 100\% do efluente no meio de cultura, utilizando as condições ótimas de luminosidade e pH definidas nas etapas anteriores. Na última fase do processo, cultivou-se a Dunaliella tertiolecta BUTCHER com as condições 
determinadas para a otimização do crescimento celular com o propósito de produção de lipídios e biorremediação do efluente (Tabela 1).

Tabela 1: Etapas para avaliação das condições de cultivo.

\begin{tabular}{|c|c|c|c|}
\hline & Exposição diária a luminosidade (horas) & $\mathrm{Ph}$ & Suplementação de UASB (\%) \\
\hline \multirow{4}{*}{$\begin{array}{l}\text { Etapa } \\
1\end{array}$} & 6 & 7,23 & - \\
\hline & 12 & 7,23 & - \\
\hline & 18 & 7,23 & - \\
\hline & 24 & 7,23 & - \\
\hline \multirow{5}{*}{$\begin{array}{l}\text { Etapa } \\
2\end{array}$} & \multirow{5}{*}{$\begin{array}{l}\text { Exposição ótima diária à luminosidade } \\
\text { determinada na Etapa } 1\end{array}$} & 5,5 & - \\
\hline & & 6,5 & - \\
\hline & & 7,23 & - \\
\hline & & 8,5 & - \\
\hline & & 9,5 & - \\
\hline $\begin{array}{l}\text { Etapa } \\
3\end{array}$ & $\begin{array}{l}\text { Exposição ótima diária à luminosidade } \\
\text { determinada na Etapa } 1\end{array}$ & $\begin{array}{l}\text { pH ótimo determinado na } \\
\text { Etapa } 2\end{array}$ & $\begin{array}{l}0 \\
25 \\
50 \\
75 \\
100\end{array}$ \\
\hline $\begin{array}{l}\text { Etapa } \\
4\end{array}$ & $\begin{array}{l}\text { Exposição ótima diária à luminosidade } \\
\text { determinada na Etapa } 1\end{array}$ & $\begin{array}{l}\text { pH ótimo determinado na } \\
\text { Etapa } 2\end{array}$ & $\begin{array}{l}\text { Suplementação ótima } \\
\text { determinada na Etapa } 3\end{array}$ \\
\hline
\end{tabular}

Para a determinação das melhores condições de cultivo, observou-se as curvas de crescimento em cada fase em conjunto com os parâmetros cinéticos. Ambos calculados através de contagens em câmaras de Neubauer realizadas diariamente com auxílio do microscópio óptico para determinação de número de células por mililitro (células. $\mathrm{mL}^{-1}$ ), como visto na Figura $1 \mathrm{~A}$, sendo avaliada a concentração celular desde o dia de inoculação (0 hora) até o final do período de 192 horas. Os parâmetros cinéticos foram velocidade específica máxima de crescimento $\left(\mu_{\text {máx }}\right)$ e o tempo de geração $\left(t_{\mathrm{g}}\right)$, determinados pelas Equações 1 e 2 , respectivamente.

$$
\ln (x)=\mu_{\text {máx }}\left(t-t_{i}\right)+\ln \left(x_{i}\right)
$$

Eq 1

Onde: $x$ - Concentração celular (células $\mathrm{mL}^{-1}$ ); $\mu_{\text {máx }}$ - Velocidade específica máxima de crescimento $\left(h^{-1}\right)$; $t_{i}$ - Tempo inicial referente a fase exponencial $(h) ;$ $\mathrm{t}-$ Tempo final referente à fase exponencial $(\mathrm{h})$; $\mathrm{x}_{\mathrm{i}}$ - Concentração celular inicial (células $\mathrm{mL}^{-1}$ ).

$$
\mathrm{t}_{\mathrm{g}}=\frac{\ln (2)}{\mu_{\text {máx }}}=\frac{0,693}{\mu_{\text {máx }}}
$$

Eq 2

Onde:

$t_{\mathrm{g}}$ - Tempo de geração $(h)$; $\mu_{\text {máx }}$ - Velocidade específica máxima de crescimento $\left(h^{-1}\right)$.

Sendo a velocidade máxima de crescimento obtida a partir da linearização dos pontos das horas iniciais do cultivo, logo após a fase de adaptação, onde os microrganismos possuem substrato suficiente para se desenvolverem de forma plena, alcançando velocidades maiores durante esse período (HISS, 2001).

\section{Extração da biomassa e avaliação do potencial de biorremediação}

Para as análises de produtividade da biomassa foram coletadas amostras do substrato no primeiro e último dia de cultivo. Posteriormente centrifugadas a $4000 \mathrm{rpm}$ durante $8 \mathrm{~min}$ em duas etapas: primeiramente para acumular a concentração da biomassa, e em seguida a centrifugação com solução de 
ácido clorídrico $(\mathrm{pH}=5)$ para retirada da salinidade do meio de cultura. Posteriormente, foram utilizadas cápsulas de porcelana para secagem das amostras em estufa a $55^{\circ} \mathrm{C}$ até atingir peso constante. A produtividade $\left(\mathrm{gL}^{-1} \mathrm{~d}^{-1}\right)$ foi determinada por meio da Equação 3.

$$
\text { Produtividade }=\frac{\text { biomassa seca }_{\text {final }} \text {-biomassa } \text { seca }_{\text {inicial }}}{\text { tempo de cultura }}
$$

Visando avaliar o potencial de biorremediação da microalga em estudo, considerou-se os cultivos acrescidos do efluente de reator UASB, fazendo-se a determinação da Demanda Química de Oxigênio (DQO), no primeiro e último dia de cultivo, conforme metodologia descrita pelo Standard Methods for the Examination of Water and Wastewater (APHA et al., 2012).

\section{Quantificação do teor lipídico}

O teor de lipídios contido na microalga está diretamente associado à produção de biocombustíveis, portanto, estendeu-se o volume dos fotobiorreatores para 10L, fazendo suplementação do cultivo com meio de cultura e efluente de UASB, observando o desenvolvimento algal e obtendo-se $2 \mathrm{~g}$ de biomassa seca através dos processos de centrifugação e secagem descritos anteriormente para posterior utilização no processo de extração.

Os lipídios foram determinados por meio da metodologia descrita por Folch et al. (1957), que consiste em submeter as amostras à extração por meio de uma mistura de clorofórmio e metanol (2:1) seguida de evaporação do solvente em estufa a 105ํC. Devido à resistência da parede celular das microalgas, a metodologia foi adaptada e a esta introduziu-se uma etapa de rompimento celular com o uso de banho ultrassônico durante 20 minutos.

O teor de lipídios foi determinado pela Equação 4.

$$
\% \text { Lipídio }=\frac{\text { volume }_{\text {fase inf }} \times\left(\text { massa }_{\text {final }}-\text { massa }_{\text {inicial }}\right)}{5 \times M}
$$

Onde: volume $_{\text {fase inf }}-$ Volume do extrato da fase inferior; massa $_{\text {final }}$ - Massa final do béquer com lipídio; massainicial - Massa do béquer seco; M - Massa da amostra.

\section{Análise dos dados}

Para o estudo da interdependência entre os parâmetros analisados, utilizou-se a matriz de correlação, em que é adotado um intervalo de valores entre -1,0 e 1,0. Quanto mais próximo de 1,0, considerando-se seu valor em módulo, maior é a correlação entre os fatores. Quando o sinal se apresenta negativo, indica que uma variável diminui com o aumento da outra, já o sinal positivo representa que uma cresce quando a outra também o faz.

Segundo Hinkle et al. (2003), correlações entre 0 e 0,30 são consideradas muito fracas; entre 0,30 e 0,50 fracas; entre 0,50 e 0,7 moderadas; entre 0,7 e 0,9 fortes; e entre 0,9 e 1 muito fortes. Além disso, foi efetuado um estudo estatístico multivariado dos dados por meio da Análise em Componentes Principais 
(ACP), a fim de estudar a formação dos grupos de variabilidades equivalentes. Para estas análises foi utilizado o software R (R CORE TEAM, 2019).

\section{RESULTADOS E DISCUSSÃO}

As curvas de concentração celular da Dunaliella tertiolecta em meio F/2 sob diferentes intervalos de exposição diária à luminosidade estão apresentadas na Figura 2.

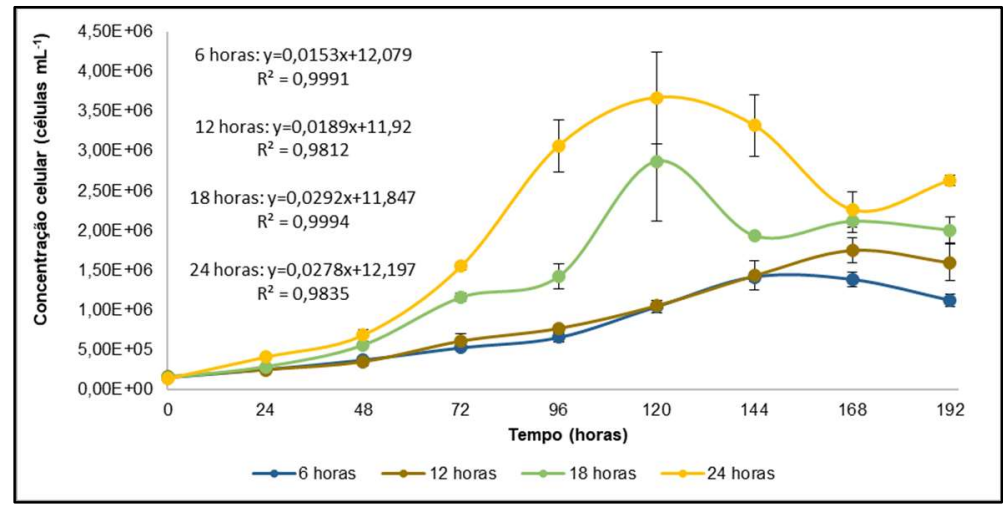

Figura 2: Curvas de concentração celular da microalga Dunaliella tertiolecta em função do tempo de cultivo para diferentes variações de luminosidade e suas respectivas equações para determinação de $\mu_{\text {máx }}$

Nota-se que a concentração celular aumenta de acordo com o intervalo de exposição, apresentando melhores resultados no cultivo submetido há 24 horas diárias de iluminação, alcançando $3,67 \times 10^{6}$ células. $\mathrm{mL}^{-}$ ${ }^{1}$ no período de 120 horas. Os cultivos de 18 e 24 horas foram os que obtiveram maiores fases log, onde o número de células de ambos começaram a decair a partir de 120 de horas. No intervalo de exposição diária de 12 horas de luminosidade, observa-se o crescimento máximo em 168 horas, atingindo a concentração $1,75 \times 10^{6}$ células. $\mathrm{mL}^{-1}$. Para melhor avaliação dos intervalos aplicados, foram analisados os dados de velocidade específica máxima de crescimento, tempo de geração e produtividade de biomassa dos cultivos, organizados na Tabela 2.

Tabela 2: Velocidades específicas máximas, tempos de geração e produtividade de biomassa dos cultivos de Dunaliella tertiolecta em meio F/2 sob variações de luminosidade.

\begin{tabular}{llll}
\hline $\begin{array}{l}\text { Luminosidade } \\
\text { (h) }\end{array}$ & $\begin{array}{l}\text { Velocidade específica máxima de crescimento } \\
\boldsymbol{\mu}_{\text {máx }}\left(\mathbf{h}^{-\mathbf{1}}\right)\end{array}$ & $\begin{array}{l}\text { Tempo de geração } \\
(\mathbf{h})\end{array}$ & $\begin{array}{l}\text { Produtividade de biomassa (g.L- } \\
\text { 1.dia-1) }\end{array}$ \\
\hline 6 & 0,0153 & 45,30 & 0,0505 \\
12 & 0,0189 & 36,67 & 0,1078 \\
18 & 0,0292 & 23,74 & 0,0177 \\
24 & 0,0278 & 24,93 & 0,0214 \\
\hline
\end{tabular}

Os valores elevados do coeficiente de determinação $\left(R^{2}\right)$ indicam que houve um ajuste amostral satisfatório ao modelo de estudo utilizado. Observa-se que quanto maior a disponibilidade de luz, maior será a densidade celular; entretanto, o cultivo de 18 horas foi o que apresentou a maior velocidade específica máxima de crescimento e, consequentemente, o menor tempo de geração, o que significa que o cultivo necessita de 23,74 horas para dobrar a densidade inicial, porém apresenta uma produtividade insatisfatória quando comparado ao cultivo de 12 horas que apresentou produtividade de 0,1078 g. $\mathrm{L}^{-1}$.dia-1 . Resultado próximo ao encontrado na literatura, 0,12 g.L $\mathrm{L}^{-1} \cdot \mathrm{dia}^{-1}$ (GOUVEIA et al., 2009). 
As análises de Chiranjeevi et al. (2016) mostraram uma maior produtividade de biomassa algal em maiores intervalos de luz, como o de 24 horas. Esse tempo de luz não foi viável neste estudo, fato proveniente do alto desenvolvimento celular ocasionado pela exposição luminosa. As microalgas atingem um estágio de fotoinibição gerando uma maior turbidez no sistema, fazendo com que a penetração de luz seja interrompida, atenuando a produtividade microalgal.

O tempo de fornecimento de luz de 12 horas foi suficiente para suprir as necessidades das microalgas, além de apresentar uma maior produtividade e simular as condições externas, isto é, quando ocorrer o cultivo em raceway com iluminação natural, fato pretendido para produção em larga escala. Portanto, esse intervalo foi considerado o mais apropriado para o cultivo de Dunaliella tertiolecta. Com isso, foram realizados cultivos alterando o $\mathrm{pH}$ do meio, bem como mantendo o seu $\mathrm{pH}$ natural $(7,23)$. As curvas de crescimento celular podem ser observadas na Figura 3.

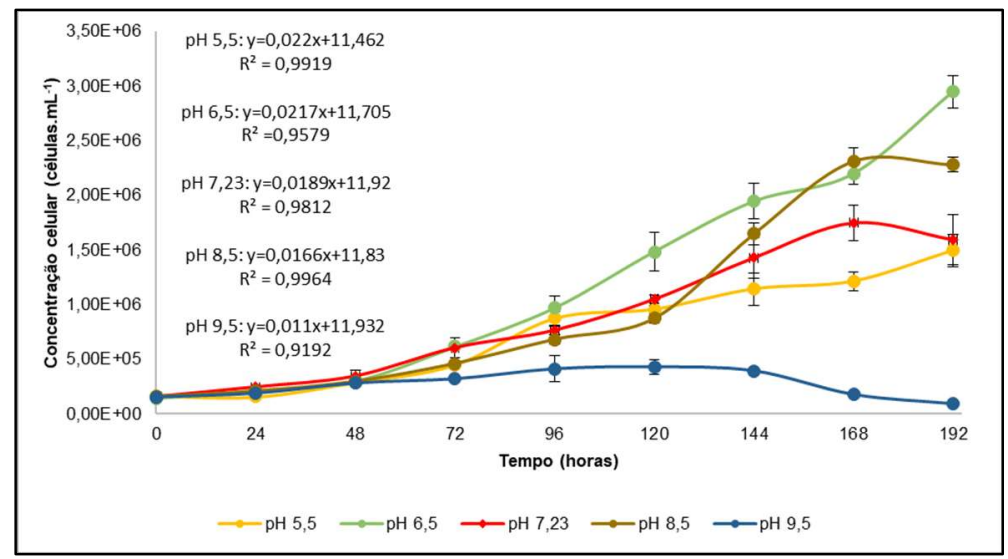

Figura 3: Curvas de crescimento celular da microalga Dunaliella tertiolecta em função do tempo de cultivo para diferentes valores de $\mathrm{pH}$ e suas respectivas equações para determinação de $\mu_{\text {máx }}$

Percebe-se que o cultivo com pH 6,5 alcançou maior concentração celular $\left(2,95 \times 10^{6}\right.$ células. $\left.\mathrm{mL}^{-1}\right)$ para um tempo de 192 horas, enquanto o de $\mathrm{pH}$ 9,5 apresentou dificuldades de desenvolvimento, alcançando apenas $4,29 \times 10^{5}$ células. $\mathrm{mL}^{-1} \mathrm{em}$ sua melhor concentração. $\mathrm{Na}$

Tabela 3 estão contidos os parâmetros cinéticos que auxiliaram na escolha do melhor pH para o cultivo da microalga.

Tabela 3: Velocidades específicas máximas de crescimento, tempos de geração e produtividade de biomassa dos cultivos de Dunaliella tertiolecta em meio $\mathrm{F} / 2$ sob variações de $\mathrm{pH}$.

\begin{tabular}{|c|c|c|c|}
\hline $\begin{array}{ll}\mathrm{pH} & \text { do } \\
\text { meio } & \end{array}$ & $\begin{array}{l}\text { Velocidade específica máxima de crescimento } \mu_{\text {máx }} \\
\left(\mathrm{h}^{-1}\right)\end{array}$ & $\begin{array}{l}\text { Tempo de geração } \\
\text { (h) }\end{array}$ & $\begin{array}{l}\text { Produtividade de biomassa (g.L- } \\
{ }^{1} . \mathrm{d}^{-1} \text { ) }\end{array}$ \\
\hline 5,5 & 0,022 & 31,51 & 0,0180 \\
\hline 6,5 & 0,0217 & 31,94 & 0,0348 \\
\hline 7,23 & 0,0189 & 36,67 & 0,1078 \\
\hline 8,5 & 0,0166 & 41,76 & 0,0190 \\
\hline 9,5 & 0,011 & 63,01 & 0,0177 \\
\hline
\end{tabular}

Os valores do coeficiente de determinação $\left(R^{2}\right)$, indicam que houve ajuste amostral satisfatório ao modelo. O cultivo de pH 5,5 apresentou maior velocidade específica e, consequentemente, menor tempo de geração. Contudo, não apresentou uma produtividade satisfatória quando comparado com o cultivo de pH 7,23, que foi cerca de seis vezes maior. Do mesmo modo, ocorreu com o pH 6,5, que apresenta uma das 
maiores densidades celulares, como observado na Figura 3, porém obteve baixa produtividade. Verificou-se também que a partir do pH 8,5 a microalga não se desenvolveu tão bem quanto os outros cultivos.

De acordo com os estudos de Ying et al. (2014) sobre a influência do pH no crescimento de Dunaliella salina, os diferentes níveis de $\mathrm{pH}$ apresentaram uma "tendência de parábola" com um valor ideal em torno de $\mathrm{pH}$ 7,0. Desse modo, observa-se que o cultivo sem alteração de pH proporcionou a melhor produtividade em biomassa, sendo esta a condição ótima de cultivo. A partir das condições de luminosidade e pH estabelecidas foi investigado a suplementação de efluente de reator UASB no meio de cultura, variando-se sua concentração entre $0,25,50,75$ e 100\%. A Figura 4 ilustra os perfis de crescimento celular da microalga nessas condições.

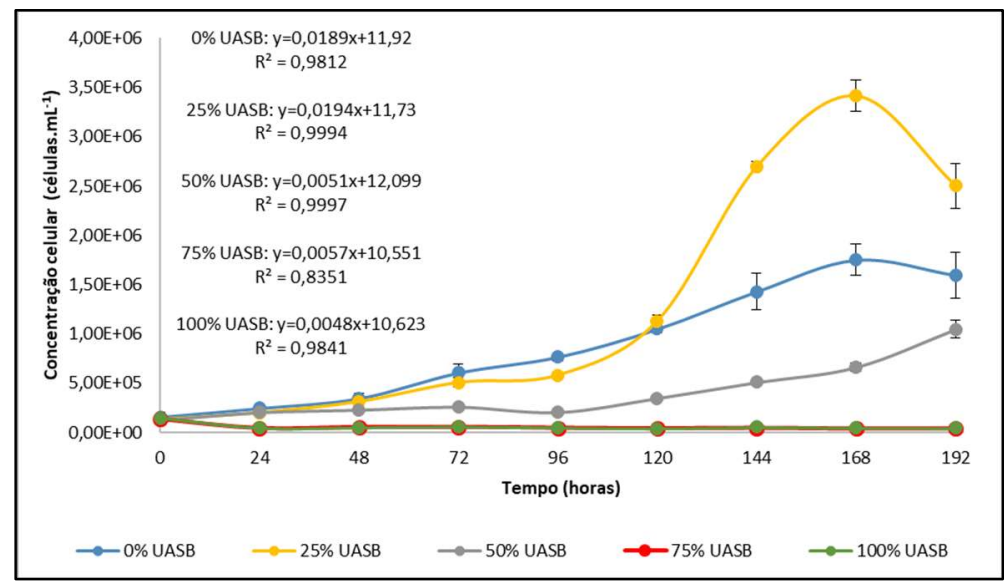

Figura 4: Curvas de crescimento celular da microalga Dunaliella tertiolecta em função do tempo de cultivo para diferentes concentrações de efluente do UASB e suas respectivas equações para determinação de $\mu_{\text {máx }}$

A Dunaliella tertiolecta exposta à concentração de $25 \%$ de efluente de reator UASB apresentou número celular inicial de $1,28 \times 10^{5}$ células. $\mathrm{mL}^{-1}$, obtendo desenvolvimento máximo em 168 horas com $3,41 \times 10^{6}$ células. $\mathrm{mL}^{-1}$. Também se faz notável o cultivo em meio puro ( $0 \%$ de efluente), que apresentou a

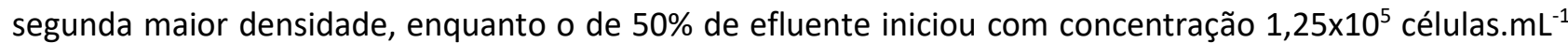
mostrando uma longa fase de adaptação ao meio, alcançando $1,05 \times 10^{6}$ células. $\mathrm{mL}^{-1}$, em 192 horas. Nos teores de 50 e $100 \%$ de efluente de reator UASB a microalga sofreu decaimento celular ao longo do tempo de análise, demonstrando que o aumento da efluente causa inibição do desenvolvimento da microalga como explicitado anteriormente. Foram realizadas verificações dos parâmetros cinéticos, assim como da remoção da DQO em todos os cultivos. Esses dados são apresentados na Tabela 4.

Tabela 4: Parâmetros avaliados nos cultivos de Dunaliella tertiolecta em diferentes concentrações de efluente de reator UASB.

\begin{tabular}{lllll}
\hline $\begin{array}{l}\text { Concentração de efluente } \\
\text { reator UASB (\%) }\end{array}$ & $\begin{array}{c}\text { deVelocidade específica } \\
\text { crescimento } \boldsymbol{\mu}_{\text {máx }}\left(\mathbf{h}^{-1}\right)\end{array}$ & $\begin{array}{c}\text { máxima } \\
\text { geração (h) }\end{array}$ & $\begin{array}{c}\text { deTempodutividade de biomassaRemoção } \\
\left(\mathbf{g . l}^{-1} \cdot \mathbf{d}^{-\mathbf{1}}\right)\end{array}$ & $\begin{array}{c}\text { de } \\
\text { DQO (\%) }\end{array}$ \\
\hline 0 & 0,0189 & 36,7 & 0,107833 & - \\
25 & 0,0194 & 35,73 & 0,034083 & 40,23 \\
50 & 0,0061 & 113,63 & 0,012083 & 36,33 \\
75 & 0,0057 & 121,6 & 0,012167 & 19,35 \\
100 & 0,0048 & 144,41 & 0,003166 & 25,35 \\
\hline
\end{tabular}

Verifica-se que a Dunaliella tertiolecta reduz sua fração pela qual a população cresce em unidade de tempo nos primeiros dias de inóculo à medida que se aumenta a concentração do efluente de reator UASB, 
fato evidenciado através da redução do fator velocidade específica, bem como o aumento do tempo de geração e redução da produtividade de biomassa.

Liu et al. (2018) avaliaram que sob uma concentração de salinidade de $30 \mathrm{ppt}$, os valores de densidade ideal de células referente aos tratamentos de águas residuais municipais foram muito superiores aqueles obtidos em concentrações de salinidade de 20 e 40 ppt, evidenciando a existência de uma concentração mais favorável. O discreto desenvolvimento da Dunaliella tertiolecta quando submetida a maiores concentrações de efluente de reator UASB, pode ser devido à baixa concentração de sal, pois, ao passo que se reduziu a quantidade do meio de cultura $F / 2$, que possui alta salinidade, aumentou-se a concentração de efluente, resultando na inadaptabilidade da alga.

A influência negativa do efluente sobre a produtividade ainda pode ser explicada pela diluição dos micronutrientes presentes no meio sintético e necessários para o desenvolvimento microalgal. Shaker et al. (2017) relata que o crescimento da biomassa microalgal ocasiona aumento da produção lipídica e isso está intimamente ligado a alterações da concentração de nutrientes presentes no meio que desempenham um papel fundamental, como o enxofre, ferro e manganês, corroborando com os resultados obtidos neste estudo.

Mulbry et al. (2008), em seus estudos, afirmam que as microalgas são utilizadas para o tratamento de águas residuais por promoverem sua purificação através do consumo de contaminantes e nutrientes solúveis que estão presentes em maiores quantidades nesses efluentes. Nesse estudo, observa-se que a melhor remoção $(40,23 \%)$ da carga poluidora ao final do cultivo foi obtida no tratamento contendo $25 \%$ de efluente de reator UASB (Tabela 1).

A partir das duas condições ótimas de cultivo: meio $\mathrm{F} / 2$ puro com 12 horas de exposição a luz e pH de 7,23; meio suplementado com UASB em $25 \%$ de proporção com 12 horas de iluminação e pH natural do meio, foram avaliadas as suas respectivas produções lipídicas, verificado para a primeira situação uma porcentagem de lipídios de $10,64 \%$ e $8,13 \%$ na segunda.

Resultados semelhantes foram encontrados nas análises de Fré et al. (2014), a Dunaliella tertiolecta quando cultivada durante $210 \mathrm{~h}$, com exposição luminosa de $12 \mathrm{~h} /$ dia e no mesmo meio utilizado no nesse estudo, apresentou o teor lipídico de 10,21 £0,03\%. Já Mata et al. (2010) relatam que a porcentagem lipídica para a $D$. tertiolecta variou entre 16 e $71 \%$. Comparando-se com a literatura citada, o decaimento da fração lipídica no cultivo com adição de efluente de UASB se justifica pela diluição dos sais e aumento da carga orgânica, fazendo com que o meio não apresente salinidade propícia para o desenvolvimento da Dunaliella tertiolecta em termos de biomassa.

\section{Análise estatística}

A fim de verificar o nível de relação entre os parâmetros avaliados para cada experimento, foi realizado o estudo da matriz de correlação dos mesmos. Por seu turno, foi utilizado a Análise de Componentes Principais (ACP) para identificar a formação de grupos que apresentam afinidades equivalentes. 
A Tabela 5 apresenta a matriz de correlação para os experimentos sob variação de luminosidade. Verifica-se correlações muito fortes entre a luminosidade diária com a velocidade específica máxima e o tempo de geração. À medida que há exposição do cultivo a luz, a velocidade específica tende a aumentar, evidenciando uma correlação positiva. Em contrapartida, o tempo de geração diminui à medida que a luminosidade aumenta, neste caso, uma correlação negativa.

Tabela 5: Matriz de correlação para a Dunaliella tertiolecta sob variações de luminosidade.

\begin{tabular}{|c|c|c|c|c|c|c|c|}
\hline & & Luminosidade & $\begin{array}{l}\text { Velocidade } \\
\text { crescimento }\end{array}$ & específica & máxima & $\begin{array}{l}\text { deTempo } \\
\text { geração }\end{array}$ & ${ }^{\text {de }}$ Produtividade \\
\hline Luminosidade & & 1 & & & & & \\
\hline $\begin{array}{l}\text { Velocidade específica } \\
\text { crescimento }\end{array}$ & máxima & $\mathrm{de}_{0,9117}$ & 1 & & & & \\
\hline Tempo de geração & & $-0,9325$ & $-0,9907$ & & & 1 & \\
\hline Produtividade & & $-0,5500$ & $-0,6847$ & & & 0,5837 & 1 \\
\hline
\end{tabular}

Fato pertinente ao objetivo do estudo, uma vez que quanto menor o tempo de geração mais rapidamente o número inicial de células é duplicado, o que aumenta a velocidade específica máxima, explicando a correlação negativa entre eles. Por outro lado, a produtividade apresentou correlação moderada e negativa com as variáveis luminosidade e velocidade específica de crescimento, mostrando uma relação inversamente proporcional. Conjuntura explicada pelo processo de fotoinibição que acontece em longos intervalos de iluminação especificamente nos estudos de 18 e $24 \mathrm{~h}$ de luz diária, como já foi dito anteriormente. Além disso, ainda apresenta correlação positiva com o tempo de geração, embora moderada, logo, são estatisticamente dependentes, sendo uma resposta ao intervalo de luminosidade diária.

Em relação à ACP para a exposição de luminosidade diária (Figura 5), observa-se que a projeção de variação dos dados nos dois eixos principais, explicam aproximadamente 97,64\% da variabilidade do processo. O fator principal 1 apresenta 83,96\% de correlação positiva entre velocidade específica máxima de crescimento com luminosidade. Entretanto, o mesmo fator demonstra uma correlação negativa da luminosidade com produtividade e tempo de geração, corroborando com os resultados apresentados na matriz de correlação.

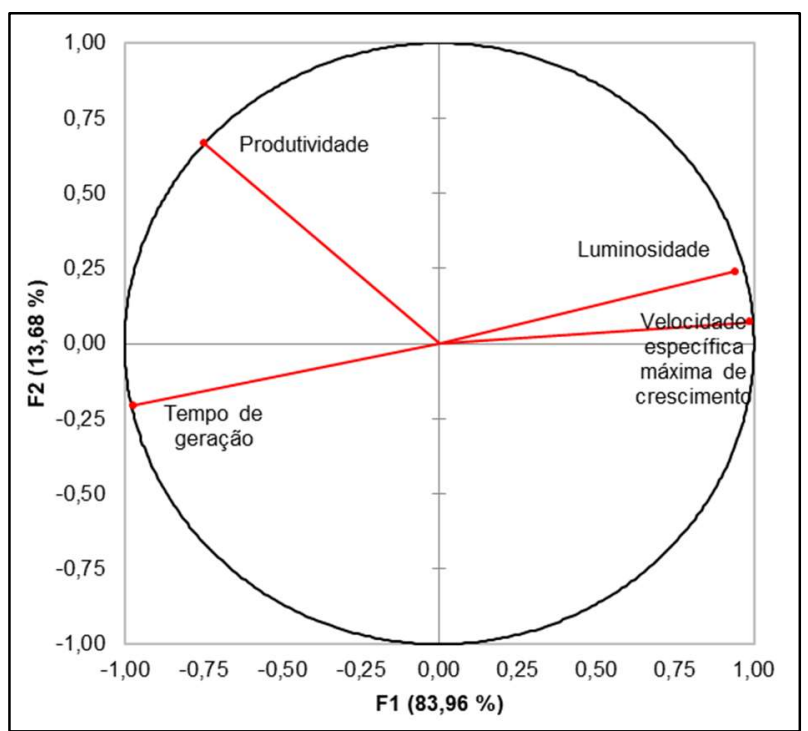

Figura 5: Análise em Componentes Principais para Dunaliella tertiolecta sob variações de luminosidade. 
A Tabela 6 apresenta a matriz de correlação referente as variações de pH para os parâmetros cinéticos. Diante dos resultados apresentados, o pH apresentou correlação muito forte positivamente com o tempo de geração e negativa com a velocidade específica máxima de crescimento. À medida que o pH do meio aumenta o tempo de geração aumenta proporcionalmente, sendo assim, quanto menor o $\mathrm{pH}$, mais satisfatório é o processo de desenvolvimento das microalgas em estudo, pois menor será o tempo de geração para se duplicar o número de células. Em contrapartida, o pH do meio é inversamente proporcional a velocidade específica máxima de crescimento, ou seja, enquanto o $\mathrm{pH}$ do meio aumenta a velocidade específica máxima de crescimento diminui.

Tabela 6: Matriz de correlação para a Dunaliella tertiolecta sob variações de $\mathrm{pH}$.

\begin{tabular}{|c|c|c|c|c|c|c|c|}
\hline & & $\mathrm{pH}$ do meic & $\begin{array}{l}\text { Velocidade } \\
\text { crescimento }\end{array}$ & específica & máxima & $\begin{array}{l}\text { deTempo } \\
\text { geração }\end{array}$ & $\mathrm{de}_{\text {Produtividade }}$ \\
\hline pH do meio & & 1 & & & & & \\
\hline $\begin{array}{l}\text { Velocidade específica } \\
\text { crescimento }\end{array}$ & máxima & $\mathrm{de}_{-0,9543}$ & 1 & & & & \\
\hline Tempo de geração & & 0,8955 & $-0,9823$ & & & 1 & \\
\hline Produtividade & & $-0,1273$ & 0,1983 & & & $-0,2688$ & 1 \\
\hline
\end{tabular}

A ACP para os experimentos sob variação de $\mathrm{pH}$, exibida na Figura 6, apresenta uma variação dos dados nos dois eixos principais que explicam aproximadamente 97,5\% da variabilidade do processo. Em relação ao fator principal 1 na qual explica 73,73\%, observa-se a formação de uma correlação muito forte e positiva entre $\mathrm{pH}$ do meio de cultura e tempo de geração, bem como uma correlação negativa do pH com a velocidade específica máxima de crescimento.

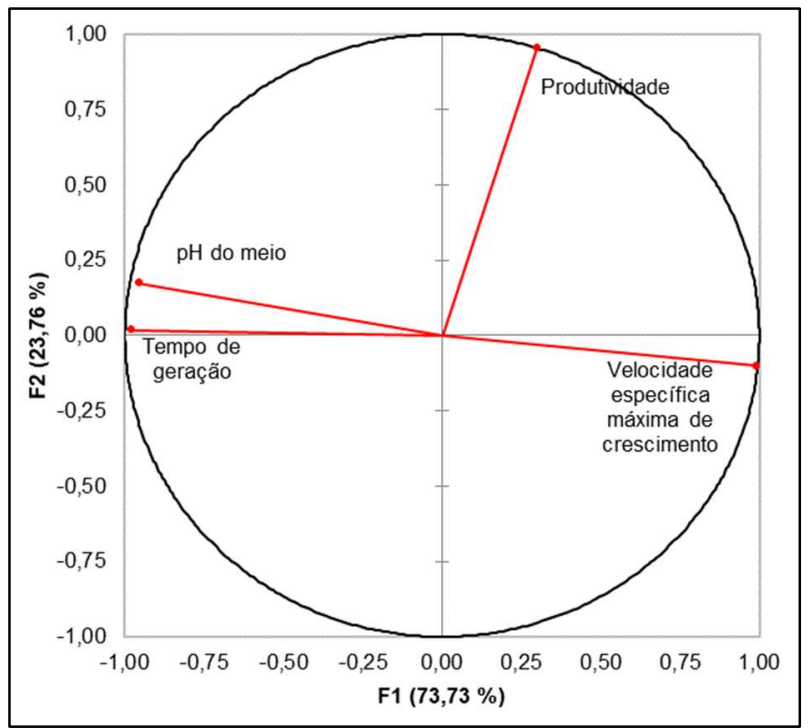

Figura 6: Análise em Componentes Principais para Dunaliella tertiolecta sob variações de pH.

Para os experimentos sob variação na concentração de efluente de reator tipo UASB (Tabela 7), constata-se de modo geral que todos os parâmetros apresentaram correlação forte, sendo a velocidade específica máxima de crescimento, a remoção de DQO e a produtividade inversamente proporcionais à porcentagem de suplementação de efluente. Fato corroborado pelo difícil desenvolvimento das células em meios com baixa salinidade, como já citado. Por outro lado, a concentração de efluente UASB apresentou correlação diretamente proporcional com o tempo de geração. 
Tabela 7: Matriz de correlação para a Dunaliella tertiolecta sob variações na concentração de efluente do reator tipo UASB.

\begin{tabular}{|c|c|c|c|c|c|}
\hline & $\begin{array}{l}\text { Concentração } \\
\text { efluente UASB }\end{array}$ & $\begin{array}{l}\text { deVelocidade específica } \\
\text { de crescimento }\end{array}$ & $\begin{array}{r}\text { máximaTempo } \\
\text { geração }\end{array}$ & $\begin{array}{l}\text { deRemoção } \\
\text { DQO }\end{array}$ & ${ }^{d e}$ Produtividade \\
\hline $\begin{array}{l}\text { Concentração de } \\
\text { efluente UASB }\end{array}$ & 1 & & & & \\
\hline $\begin{array}{l}\text { Velocidade específica máxima de } \\
\text { crescimento }\end{array}$ & ${ }^{e}-0,8204$ & 1 & & & \\
\hline $\begin{array}{l}\text { Tempo de } \\
\text { geração }\end{array}$ & 0,9126 & $-0,9797$ & 1 & & \\
\hline $\begin{array}{l}\text { Remoção de } \\
\text { DQO }\end{array}$ & $-0,8343$ & 0,7339 & $-0,7625$ & 1 & \\
\hline Produtividade & $-0,9085$ & 0,9681 & $-0,9959$ & 0,7093 & 1 \\
\hline
\end{tabular}

A ACP para os experimentos sob variação de concentração de efluente de reator UASB, ilustrada na Figura 7, apresenta uma variação nos dois eixos principais que explicam aproximadamente $97,09 \%$ da variabilidade do processo. Assim como apresentado na matriz de correlação, no estudo de ACP, a concentração de UASB também apresentou forte correlação positiva com o tempo de geração, o que pode ser explicado pelo fato de que pequenas concentrações de UASB permitiram um tempo de geração da duplicação das células menor, o que foi ideal para o processo. Por outro lado, à medida que a concentração do efluente UASB aumentava, o tempo de geração se elevava proporcionalmente, demonstrando uma afinidade diretamente proporcional entre eles dentro do processo.

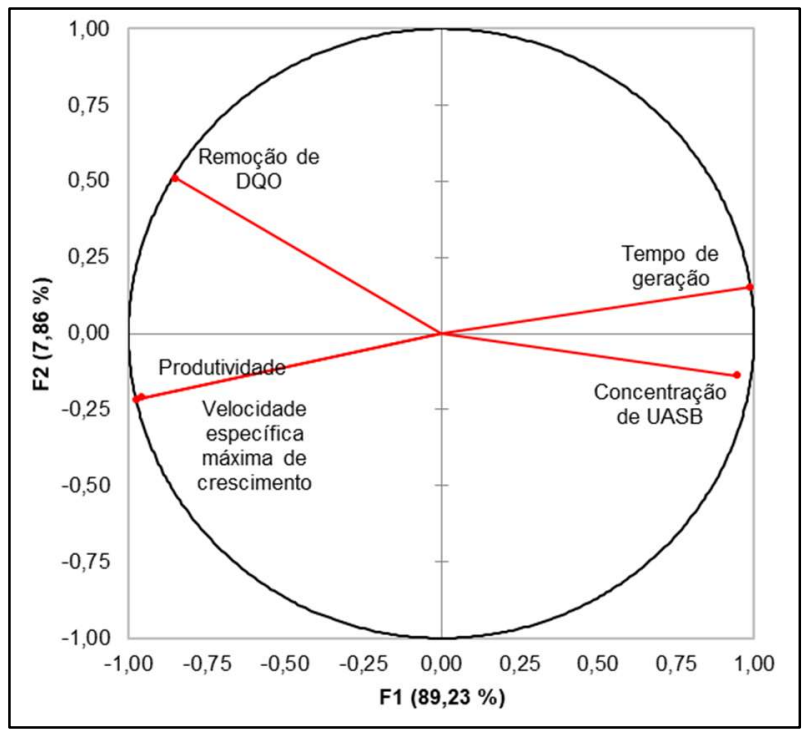

Figura 7: Análise em Componentes Principais para Dunaliella tertiolecta sob variações na concentração de efluente do UASB.

Em relação aos demais parâmetros avaliados comparados com a concentração de efluente de UASB, os mesmos apresentaram correlações fortes e negativas. Fato este já explicado pela dificuldade do desenvolvimento das células em meios com baixa salinidade, tendo em vista que o efluente dilui os sais presentes no meio $F / 2$. Sendo assim, ao encontrar um meio adverso para seu desenvolvimento, a produtividade que depende diretamente da velocidade de crescimento para se desenvolver é prejudicada dentro do processo, no qual resulta em uma baixa remoção de DQO, já que a mesma também possui uma relação direta com a produtividade para exercer a remediação. 


\section{CONCLUSÕES}

Com os resultados obtidos no presente estudo conclui-se que: As melhores condições para o desenvolvimento da microalga Dunaliella tertiolecta BUTCHER foi no tempo de 12 horas de iluminação diárias e $\mathrm{pH}$ natural do meio $\mathrm{F} / 2(7,23)$; A proporção de efluente resultante do tratamento anaeróbico de esgoto doméstico mais favorável para a microalga em estudo foi de $25 \%$; obteve-se $8,13 \%$ de lipídios nos cultivos com efluente UASB e os sem adição de efluente apresentaram 10,64\%.

Através das análises de DQO constatou-se o potencial de biorremediação da alga com remoção de $40,23 \%$ da carga poluidora; verificou-se que a maioria dos parâmetros apresentaram correlação forte, entretanto, a produtividade apresentou moderada correlação com os demais parâmetros; Através da ACP, observou-se a formação de grupos relacionados mais intimamente, mostrando a importância de se ter um bom planejamento dos parâmetros para que se possa otimizar a produção da biomassa da microalga.

\section{REFERÊNCIAS}

ALMEIDA, P. G. S.; RIBEIRO, T. B.; SILVA, B. S.; AZEVEDO, L. S.; CHERNICHARO, C. A. L.. Contribuição para o aprimoramento de projeto, construção e operação de reatores UASB aplicados ao tratamento de esgoto sanitário, Parte 6 : Qualidade do efluente. Revista DAE, v.66, n.214, 2018. DOI: http://doi.org/10.4322/dae.2018.043

APHA; AWWA; WPCF. American Public Health Associaton; American Water Works Associaton; Water Environment Federaton. Standard Methods for the Examinaton of Water and Wastewater. 22. ed. Washington: APHA, 2012.

CHIRANJEEVI, P.; MOHAN, S. V.. Critical parametric influence on microalgae cultivation towards maximizing biomass growth with simultaneous lipid productivity. Renewable Energy, v.98, p.1-8, 2016. DOI:

http://doi.org/10.1016/j.renene.2016.03.063

CREMONEZ, P. A.; FEROLDI, M.; ROSSI, E.; FEIDEN, A.; CAMARGO, M. P.; CREMONEZ, F. E.; KLAJN, F. F.. Biodiesel production in Brazil: Current scenario and perspectives. Renewable and Sustainable Energy Reviews, v.42, n.2015, p.415-428, 2015. DOI:

http://doi.org/10.1016/j.rser.2014.10.004

FOLCH, J.; LEES, M.; SLOANE STANLEY, G. H.. A simple method for the isolation and purification of total lipides from animal tissues. J. Biol. Chem., v.226, n.1, p.497-509, 1957.

FRANCISCO, S. A.. Recuperação de microalgas cultivadas em efluente de tratamento anaeróbio de esgoto por processo de coagulação-floculação. Vitória: Universidade Federal do Espírito Santo, 2016.

FRÉ, N. C.; RECH, R.; MARCíLIO, N. R.. Influência da luminosidade e concentração salina na produção de lipídios e carotenoides microalga Dunaliella tertiolecta em fotobiorreator airlift. In: CONGRESSO BRASILEIRO DE ENGENHARIA QUÍMICA, 20. Anais. Florianópolis, 2014. DOI: http://doi.org/10.5151/chemeng-cobeq2014-1171-20672$\underline{176720}$
GOUVEIA, L.; OLIVEIRA, A. C.. Microalgae as a raw material for biofuels production. Journal of Industrial Microbiology and Biotechnology, v.36, p.269-274, 2009. DOI: https://doi.org/10.1007/s10295-008-0495-6

GUILLARD, R. R. L.. Culture of phytoplankton for feeding marine invertebrates. In: SMITH, W. L.; CHANLEY, M. H.. Culture of marine invertebrate animals. New York: Plenum, 1975. p.29-60. DOI: http://dx.doi.org/10.1007/978-1-4615$8714-93$

HISS, H.. Cinética de processos fermentativos. In SCHMIDELL, W.; LIMA, U. A.; AQUARONE, E.; BORZANI, W.. Biotecnologia Industrial. São Paulo: Edgard Blücher Ltda., 2001. p.93-122.

HINKLE, D. E.; WIERSMA, W.; JURS, S. G.. Applied Statistics for the Behavioral Sciences. 5 ed. Boston: Houghton Mifflin, 2003.

LIU, Y.; YILDIZ, I.. The effect of salinity concentration on algal biomass production and nutrient removal from municipal wastewater by Dunaliella salina. International Journal Energy Research, p.1-10, 2018. DOI: https://doi.org/10.1002/er.3967

MASSON, I. S.; COSTA, G. H. G.; ROVIERO, J. P.; FREITA, L. A.; MUTTON, M. A.; MUTTON, M. J. R.. Produção de bioetanol a partir da fermentação de caldo de sorgo sacarino e cana-deaçúcar. Ciência Rural, v.45, n.9, p.1965-1700, 2015. DOI: http://dx.doi.org/10.1590/0103-8478cr20130549

MATA, T. M.; MARTINS, A. A.; CAETANO, N. S.. Microalgae for biodiesel production and other applications: A review. Renewable and Sustainable Energy Reviews, v.14, p.217232, 2010. DOI:

http://dx.doi.org/10.1016/j.rser.2009.07.020

MULBRY, W.; KONDRAD S.; PIZARRO, C.; KEBELDEWESTHEAD, E.. Treatment of dairy manure effluent using freshwater algae: Algal productivity and recovery of manure 
nutrients using pilot-scale algal turf scrubbers. Bioresource

Technology, v.99, p.8137-8142, 2008. DOI:

http://doi.org/10.1016/i.biortech.2008.03.073

NASCIMENTO, R. C.; FERNANDES, M. S.; SANTANA, H.; CEREIJO, C.; GARCIA, L. C.; SIQUEIRA, F. G.; BRASIL, B. S. A. F.. Avaliação do cultivo de microalgas em fotobiorreatores de placas planas para a produção de biomassa e biorremediação de efluente da agroindústria de óleo de palma. In: ENCONTRO DE PESQUISA E INOVAÇÃO DA EMBRAPA AGROENERGIA. Anais. Brasília, 2016.

NEOFOTIS, P.; HUANG, A.; SURY, K.; CHANG, W.; JOSEPH, F.; GABR, A.; TWARY, S.; QIU, W.; HOLGUIN, O.; POLLE, J. E. W. Characterization and classification of highly productive microalgae strains discovered for biofuel and bioproduct generation. Algal Research, v.15, p.164-178, 2016. DOI: https://doi.org/10.1016/i.algal.2016.01.007

PAREDES, J. R.; RAMIREZ, C. J. J.. Energías renovables variables y su contribución a la seguridad energética: complementariedad en Colombia. Colombia: Banco Interamericano de Desarrollo, 2017.
PITTMAN, J. K.; DEAN, A. P.; OSUNDEKO, O.. The potential of sustainable algal biofuel production using wastewater resources. Bioresource Technology, v.102, p.17-25, 2011. DOI: https://doi.org/10.1016/j.biortech.2010.06.035

R CORE TEAM. R: A language and environment for statistical computing. Vienna: R Foundation for Statistical Computing, 2019.

ROMERO, L.; GUEVARA, M.; ARMAS, H. D.; LODEIROS, C.. Cuantificación de carotenoides totales y $\beta$-caroteno en dos cepas de Dunaliella Salina (chlorophyta, volvocales) aisladas de lagunas hipersalinas de venezuela. Boletín del Instituto Oceanográfico de Venezuela, v.47, n.1, p.67-76, 2008.

SHAKER, S.; MOROWVAT, M. H.; GHASEMI, Y.. Effects of Sulfur, Iron and Manganese Starvation on Growth, $\beta$ carotene Production and Lipid Profile of Dunaliella salina. Journal of Young Pharmacists, v.9, n.1, p.43-46, 2017. DOI: https://doi.org/10.5530/iyp.2017.9.9

YING, K.; GILMOUR, D. J.; ZIMMERMAN, W. B.. Effects of CO2 and $\mathrm{pH}$ on Growth of the Microalga Dunaliella salina. Journal of Microbial \& Biochemical Technology, v.6, n.3, p.167-173, 2014. DOI: https://doi.org/10.4172/1948-5948.1000138

A CBPC - Companhia Brasileira de Produção Científica (CNPJ: 11.221.422/0001-03) detém os direitos materiais desta publicação. Os direitos referem-se à publicação do trabalho em qualquer parte do mundo, incluindo os direitos às renovaç̃oes, expansões e disseminações da contribuiç̃o, bem como outros direitos subsidiários. Todos os trabalhos publicados eletronicamente poderão posteriormente ser publicados em coletâneas impressas sob coordenação da Sustenere Publishing, da Companhia Brasileira de Produção Científica e seus parceiros autorizados. Os (as) autores (as) preservam os direitos autorais, mas não têm permissão para a publicação da contribuição em outro meio, impresso ou digital, em português ou em tradução. 